\title{
Draft genome sequence of the male-killing Wolbachia strain wBol1 reveals recent horizontal gene transfers from diverse sources
}

\author{
Anne Duplouy ${ }^{1,2^{*}}$, Iñaki Iturbe-Ormaetxe ${ }^{1,3}$, Scott A Beatson ${ }^{4}$, Jan M Szubert ${ }^{4}$, Jeremy C Brownlie ${ }^{1,5}$, \\ Conor J McMeniman ${ }^{1,6}$, Elizabeth A McGraw ${ }^{1,3}$, Gregory D D Hurst ${ }^{7}$, Sylvain Charlat ${ }^{8}$, Scott L O'Neill ${ }^{1,3,9}$ \\ and Megan Woolfit ${ }^{1,3^{*}}$
}

\begin{abstract}
Background: The endosymbiont Wolbachia pipientis causes diverse and sometimes dramatic phenotypes in its invertebrate hosts. Four Wolbachia strains sequenced to date indicate that the constitution of the genome is dynamic, but these strains are quite divergent and do not allow resolution of genome diversification over shorter time periods. We have sequenced the genome of the strain wBol1-b, found in the butterfly Hypolimnas bolina, which kills the male offspring of infected hosts during embyronic development and is closely related to the non-male-killing strain wPip from Culex pipiens.

Results: The genomes of WBol1-b and WPip are similar in genomic organisation, sequence and gene content, but show substantial differences at some rapidly evolving regions of the genome, primarily associated with prophage and repetitive elements. We identified 44 genes in wBol1-b that do not have homologs in any previously sequenced strains, indicating that Wolbachia's non-core genome diversifies rapidly. These wBoll-b specific genes include a number that have been recently horizontally transferred from phylogenetically distant bacterial taxa. We further report a second possible case of horizontal gene transfer from a eukaryote into Wolbachia.

Conclusions: Our analyses support the developing view that many endosymbiotic genomes are highly dynamic, and are exposed and receptive to exogenous genetic material from a wide range of sources. These data also suggest either that this bacterial species is particularly permissive for eukaryote-to-prokaryote gene transfers, or that these transfers may be more common than previously believed. The wBol1-b-specific genes we have identified provide candidates for further investigations of the genomic bases of phenotypic differences between closely-related Wolbachia strains.
\end{abstract}

\section{Background}

Wolbachia pipientis, a bacterial endosymbiont of a vast range of insect and other arthropod species [1], is maternally transmitted, and commonly enhances its transmission to the next host generation by modifying its hosts' reproductive systems. Different strains of Wolbachia induce different modifications, including parthenogenesis, feminization of genetic males, cytoplasmic incompatibility, and male-killing [2]. Other strains ensure their transmission

\footnotetext{
*Correspondence: duplouyanne@yahoo.fr; meg.woolfit@monash.edu

${ }^{1}$ School of Biological Sciences, University of Queensland, 4072, Brisbane, QLD, Australia

${ }^{3}$ School of Biological Sciences, Monash University, Clayton, VIC 3800, Australia Full list of author information is available at the end of the article
}

by becoming obligate mutualists [3], while yet others use a combination of strategies and act as moderate reproductive parasites while providing their host with benefits such as increased fecundity [4], metabolic provisioning during nutritional stress [5] or protection from pathogens [6-9].

This diversity of host effects is mirrored by the genetic diversity found between strains of Wolbachia pipientis. To date, the complete genomes of four Wolbachia strains have been sequenced and described: $w$ Mel [10], $w \mathrm{Ri}$ [11], $w$ Pip [12], and $w \mathrm{Bm}$ [13]. These four strains represent a range of phenotypes: $\mathrm{wBm}$ is an obligate mutualist, while $w \mathrm{Mel}, w \mathrm{Ri}$ and $w$ Pip induce cytoplasmic incompatibility and offer varying degrees of pathogen protection to their hosts. They also represent a moderate 
proportion of the phylogenetic diversity present in Wolbachia. This species has been divided into fourteen 'supergroups', or divergent clades, named A to M [14,15], and the complete genomes are drawn from three of these: $w \mathrm{Mel}$ and $w \mathrm{Ri}$ are $\mathrm{A}$-group, $w$ Pip $\mathrm{B}$-group, and $w \mathrm{Bm} \mathrm{D}$-group. Comparison of the four strains suggests that Wolbachia, in contrast to obligate symbionts such as Buchnera, have highly flexible gene content, despite their generally small genome sizes e.g. [16]. However, the divergence between previously sequenced strains has made it difficult to characterise the tempo and mode of divergence of Wolbachia strains. In addition, the sheer number of differences between genomes makes it impossible to link genomic differences to any particular aspect of symbiosis.

In this paper, we addressed this problem by sequencing the genome of $w$ Bol1-b from the butterfly Hypolimnas bolina. MLST phylogeny indicates that this strain is closely related to $w$ Pip, the CI-inducing strain in the mosquito Culex pipiens (Figure 1a). Comparison of the two strains can therefore give insight into divergence over short periods of time associated with symbiosis in different host species, and with different phenotypes induced in those hosts. wBol1-b induces male-killing, a phenotype observed in a range of insect symbionts, including several Wolbachia strains [17-20]. wBol1-b is also notable for very high vertical transmission efficiency, leading to high prevalence [21] that affects the behaviour and ecology of the host [22]. This has driven the evolution of suppressor genes that prevent male killing from occurring in some host populations [23]; in these populations, $w$ Bol1-b induces CI [24]. In addition to providing a view of Wolbachia divergence over short periods of time, the $w$ Boll-b genome also represents the second genome for a male-killing bacterium [25], and one for which interaction with the host can be investigated through examination of the suppression system.

This paper describes the annotated draft genome sequence of $w$ Boll-b, and compares its organization and gene content to those of four previously sequenced Wolbachia genomes. We identify and characterize genes that are specific to $w$ Bol1-b, and show that a surprising number of these genes have been recently horizontally transferred from other taxa, including two genes that may be eukaryotic in origin. The results presented here provide a useful resource for future investigations of the genetic bases of male killing in Wolbachia, and further our understanding of the role played in Wolbachia genome evolution by frequent horizontal gene transfer.

\section{Results \& discussion}

\section{Wolbachia purification and genome sequencing}

This is the first Wolbachia genome project to be completed using a combination of a novel method for

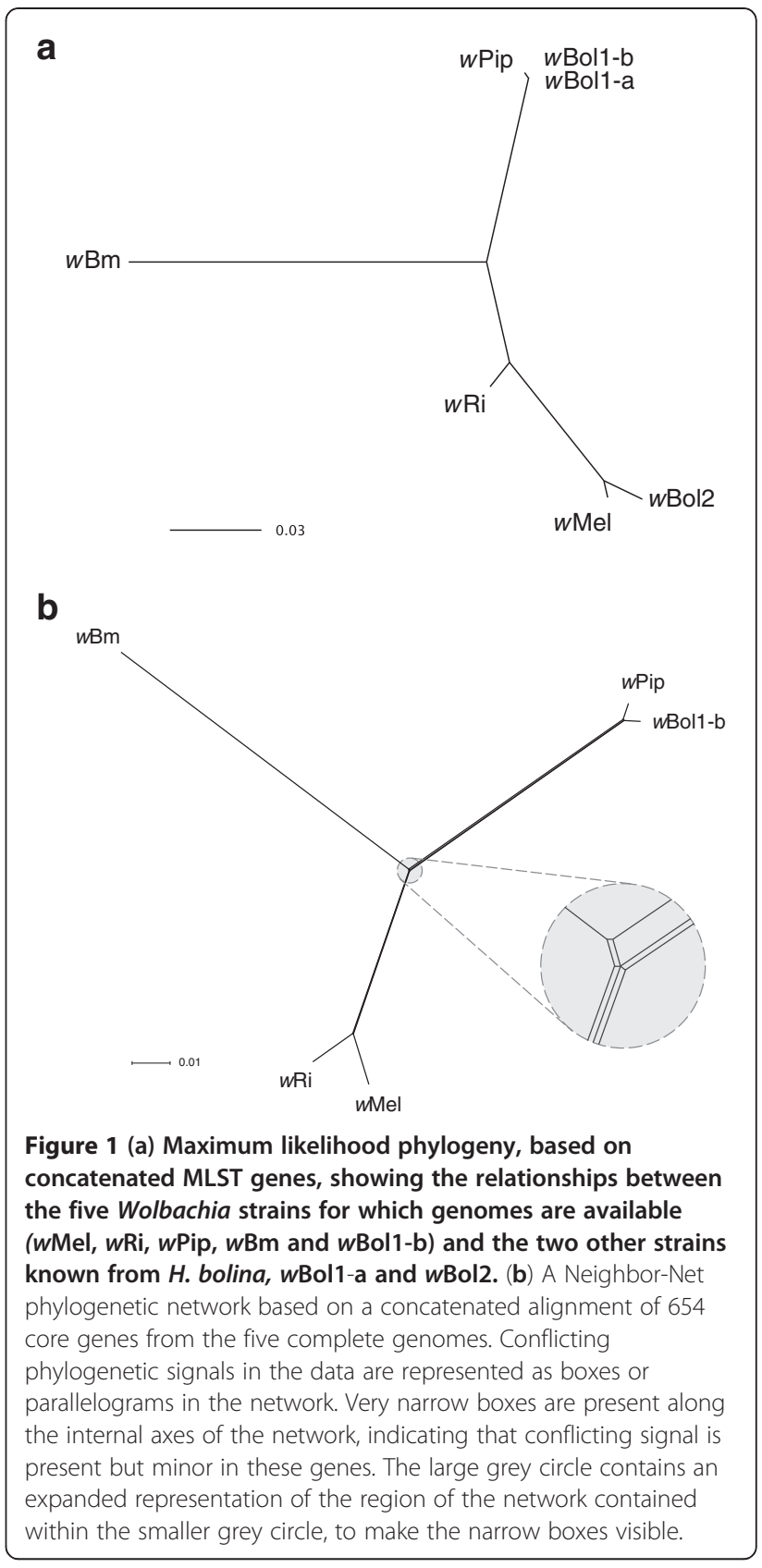

preparing sequencing material and next-generation sequencing techniques. Because the ratio of Wolbachia to host DNA is typically very low, previous Wolbachia genome projects have required extensive and laborious purifications from extremely large numbers of host individuals before sequencing. Here we have overcome this difficulty in a number of ways. First, we used a short period of cell culture to amplify Wolbachia originally isolated from a single host individual. This removed the need for maintenance of extremely large numbers of butterfly hosts, and also increased the likelihood that our sequence data represent a single clonal lineage of $w$ Bol1-b. As only a small 
number of cell culture passages were performed, it is unlikely that mutations could have arisen and fixed in the $w$ Bol1-b genome during this process, so the sequence we obtained should represent that found in the insect. Secondly, we obtained Wolbachia from host cells using a version of a simple and rapid purification method. This method produces exceptionally pure endosymbiont material for sequencing: in our wBol1-b 454 GS-FLX libraries fewer than $20 \%$ of sequence reads mapped to the host genome, while a refined version of the method can reduce this to fewer than 3\% host reads [26]. Finally, the depth of sequence coverage obtained using next-generation sequencing (approximately 70X for this project) means that it is likely that a near-complete Wolbachia genome sequence could be obtained and assembled from a single sequencing run even if far more substantial host contamination were present. The combination of refined purification methods and development of sequencing technologies should facilitate the more rapid completion of future endosymbiont genome sequencing projects.

\section{Genome content}

The draft genome of $w$ Bol1-b contains 1,418,863 nucleotides (nt) in 13 scaffolds ranging in length from 3805 to 879,879 nt. This genome shares general characteristics with those of previously sequenced Wolbachia strains, including genome size, approximate number of coding sequences, and GC content (Table 1). The scaffolds contain 1257 predicted coding sequences. We used orthoMCL [27] to identify orthologous clusters of coding genes in the genomes of $w$ Bol1-b, $w$ Pip, $w \mathrm{Mel}, w \mathrm{Ri}$, and $w \mathrm{Bm}$. We identified 654 core genes which are present in a single copy in each of the five strains, and a further 10 genes present in all strains but with a paralogous second copy (caused by a lineage-specific gene duplication) present in at least one strain (Additional file 1: Table S1). This is similar to the Wolbachia core genome size of 621 genes predicted by Ishmael et al. [16] by extrapolation from microarray-based comparative genome hybridization analyses of A-group Wolbachia strains.
To assess the completeness of our assembly, we searched for orthologous gene clusters that were present in the other four sequenced Wolbachia genomes (and were thus potentially core genes) but that were absent from the $w$ Bol1-b assembly. Only two clusters matched this pattern: a gene encoding an acetylornithine transaminase protein (WPa_0783 in $w$ Pip), and a hypothetical protein-coding gene (WPa_0114). Orthologs of both of these genes were present in the $w$ Bol1-b read data, but were on small contigs not assembled into scaffolds. We therefore believe that it is reasonable to assume that we have sequenced very close to $100 \%$ of the $w$ Boll-b genome, and that the vast majority of the non-repetitive protein-coding genes have been assembled and incorporated into scaffolds.

The genomes of the two B-group strains $w$ Pip and $w$ Bol1-b contain similar sets of genes likely to be involved in host interactions, including genes encoding membrane proteins and secretion systems. The $w$ Bol1-b genome contains the 14 genes coding for the proteins that make up the Wolbachia type four secretion system (T4SS) $[10,28]$. As in the four other fully sequenced Wolbachia strains, 11 of the wBol1-b T4SS genes are grouped into two operons (the first including virB4, $\operatorname{vir} B 3$ and four copies of virB6, and the second virB8, $\operatorname{virB9}$, virB10, virB11 and $\operatorname{virD4}$ ), while the second copies of virB4, virB8 and virB9 are distributed elsewhere in the genome.

Ankyrin (ANK) repeat domains are abundant in Wolbachia genomes $[10,29,30]$, and may be involved in interacting with or manipulating the host e.g. [31,32]. A total of 61 ANK coding genes were found in the $w$ Bol1b draft genome, one more ANK coding gene than in $w$ Pip [30], and substantially more than the two A group strains previously sequenced, $w \mathrm{Mel}$ and $w \mathrm{Ri}$, which have 23 and 35 ANK genes respectively (Table 1 ). Despite the similar number of ANK genes in $w$ Pip and $w$ Bol1-b, not all are orthologous between the two strains: eight of the $w$ Bol1-b ANK genes were not grouped into ortholog clusters with $w$ Pip members. Several of these genes have been lost or pseudogenised in the $w$ Pip lineage after

Table 1 General characteristics of Wolbachia genome sequences

\begin{tabular}{|c|c|c|c|c|c|}
\hline Strain & wBol1-b scaffolds & $w P i p$ & wMel & $w \mathrm{Ri}$ & $w B m$ \\
\hline Supergroup & B & B & A & A & D \\
\hline Genome size (nt) & $1,418,863$ & $1,482,455$ & $1,267,782$ & $1,445,873$ & $1,080,084$ \\
\hline $\mathrm{G}+\mathrm{C} \%$ & 33.9 & 34.2 & 35.2 & 35.2 & 34.2 \\
\hline CDSs & 1257 & 1386 & 1196 & 1150 & 903 \\
\hline tRNAs & 34 & 34 & 34 & 34 & 34 \\
\hline rRNAs & 1 each of $5 S, 16 S, 23 S$ & 1 each of $5 S, 16 S, 23 S$ & 1 each of $5 S, 16 S, 23 S$ & 1 each of $5 S, 16 S, 23 S$ & 1 each of $5 S, 16 S, 23 S$ \\
\hline ANKs & 61 & 60 & 23 & 35 & 5 \\
\hline Genome accession \# & $\mathrm{CAOH} 01000001-\mathrm{CAOH} 01000144$ & NC_010981 & NC_002978 & NC_012416 & NC_006833 \\
\hline
\end{tabular}


divergence from $w$ Bol1-b, while others appear to have been newly introduced into the $w$ Boll-b genome.

\section{Genome recombination and rearrangement}

MLST data had previously indicated that $w$ Bol1-b and $w$ Pip were closely related (Figure 1a). A phylogenetic network analysis (Figure 1b) based on the concatenated alignments of the 654 core genes from the five genomes confirms that these are the most closely related strains sequenced to date. Boxes or parallelograms in the network indicate conflicting phylogenetic signal [33]; this network contains only very narrow boxes, indicating that there is a low level of conflict in the data at the nucleotide level. This is perhaps somewhat unexpected, given the high levels of recombination previously reported to occur between Wolbachia genomes e.g. [11], but there is a simple explanation: it is likely that most homologous recombination occurs between closely related strains, and thus within supergroups. As we have data from only one or two strains per supergroup, this network is unlikely to capture the signal of the majority of homologous recombination that may be occurring between Wolbachia genomes. Our network does suggest, however, that homologous recombination of core genes between supergroups is not rampant.

Previous comparative analyses of $w \mathrm{Mel}, w \mathrm{Ri}, w$ Pip and $w \mathrm{Bm}$ have shown a relatively high level of genome rearrangement between these four Wolbachia strains $[12,13]$. In contrast, large regions of the $w$ Bol1-b genome are almost perfectly colinear with their corresponding regions in $w$ Pip (Figure 2). Scaffolds 2 and 20 cover approximately $82 \%$ of the $w$ Boll-b genome, and show high syntenic conservation with $w$ Pip, with the notable exceptions of one large inversion in each scaffold. The largest inversion in scaffold02 is approximately $156,400 \mathrm{nt}$ long and contains 171 coding sequences (CDSs, from wBol1_0654 to wBol1_0835), while the largest inversion in scaffold20 is approximately $93,000 \mathrm{nt}$ and contains 82 CDSs (from wBol1_1153 to wBol1_1242).

Wolbachia genomes are extremely rich in insertion sequences (IS) with transpositional activity [34]. These elements are often associated with junctions between syntenic blocks $[11,35]$. Three of the four junctions of the two major inversions described above are associated with a copy of an IS2 transposase in $w$ Pip (WPa_0116, WPa_0846 and WPa_1024). This transposase is present in 46 annotated copies in the $w$ Pip genome, 44 of them identical. The $w$ Bol1-b assembly contains one degenerate copy of this transposase, and there are at least two other moderately diverged but complete copies present in short contigs not included in the assembly. The lack of sequence divergence between the different copies of this transposase gene in $w$ Pip suggests that it may be active and involved in ongoing transpositional activity in both $w$ Bol1-b and $w$ Pip.

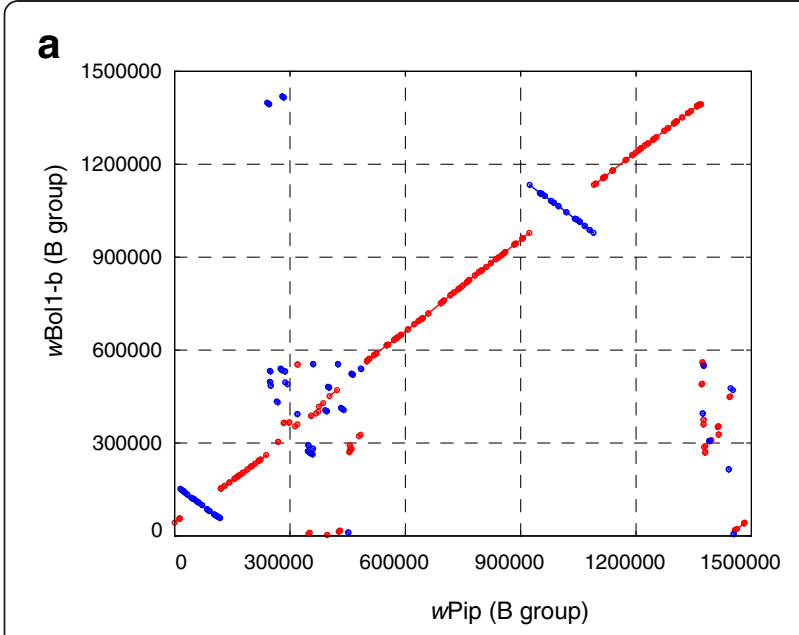

b

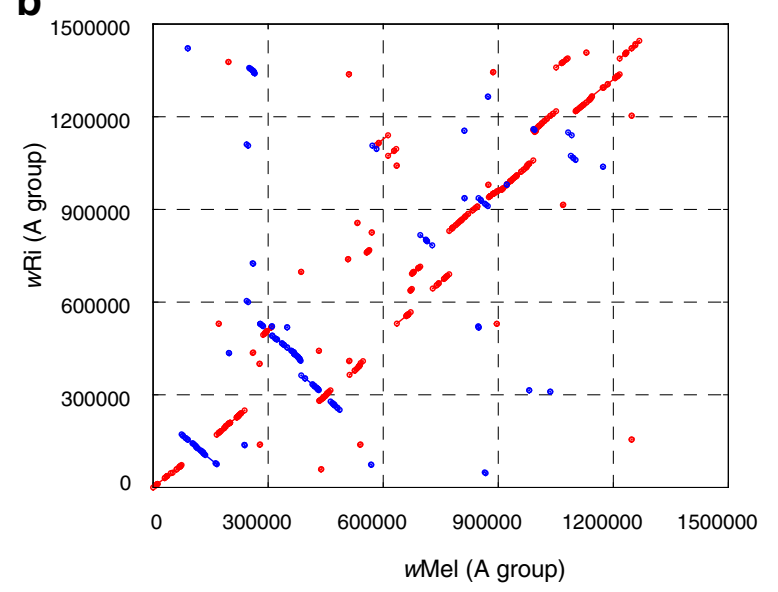

C

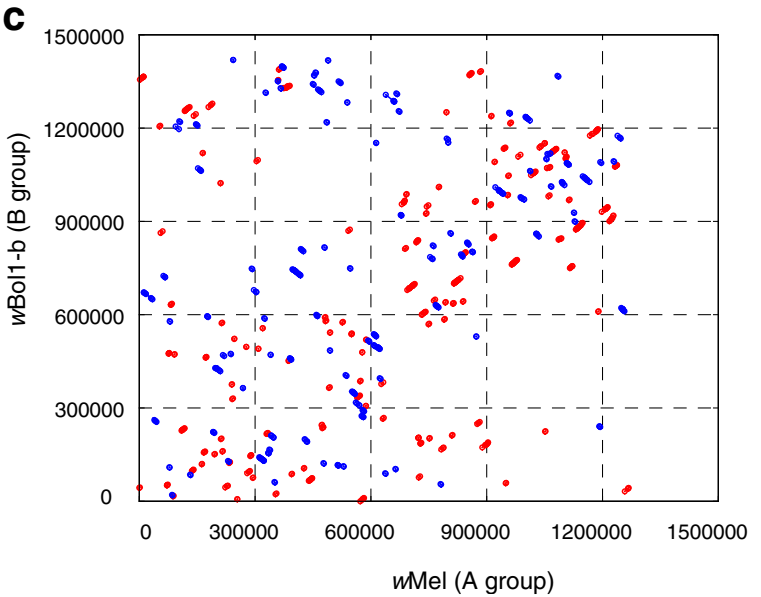

Figure 2 Dot plots showing differences in syntenic conservation between Wolbachia genomes. Dots and lines represent unique genomic sequence matches, red for a forward match and blue for a reverse match (inversions). Comparisons are shown between the genomes of (a) wBol1-b and wPip, the two complete B-group genomes, (b) wMel and wRi, the two complete A-group genomes, and (c) wBol1-b and $w \mathrm{Mel}$, a representative comparison between A- and B-group genomes. Numbers along the axes represent genome coordinates. 


\section{WO prophage and wBol1-b-specific genes}

Each of the previously sequenced A- and B-group Wolbachia genomes contains between two and five prophage $W O$ copies. The draft $w$ Bol1-b assembly currently contains nine fragmentary prophage regions, many of which are short and associated with the end of scaffolds. This suggests that these regions are incompletely assembled prophages, and that the true number of prophage copies in the genome is substantially smaller. The longest prophage region, and probably the only one completely assembled, consists of the complete length of scaffold 17, from wBol1_0161 to wBol1_0219. This region is almost precisely colinear with the $w$ Pip prophage WOPip5, as defined in [36]. Colinearity is disrupted only by a small number of insertion or deletion events, each affecting between one and five genes. The full list of putative prophage regions in the assembly is given in Additional file 2: Table S3.

Many horizontal gene transfer events into Wolbachia are likely to be mediated by bacteriophage, which are known to transfer laterally between Wolbachia strains coinfecting the same host [37] and are capable of transferring flanking non-phage genes in the process [38], thus facilitating horizontal gene transfer and genome diversification. To identify possible recent horizontal gene transfers into the $w$ Bol1-b genome, we used $w$ Bol1- $\mathrm{b}$ genes that had not been clustered with any other gene in the orthoMCL analysis (and were therefore putatively $w$ Bol1-b-specific) as blastp queries against the NR database. 26 of these genes had blastp matches to genes from other Wolbachia strains not included in the clustering analysis, and are thus components of the accessory genome but are not $w$ Bol1-b-specific. A total of 44 genes are present in wBol1-b but no other currently sequenced Wolbachia strain (Additional file 2: Table S4). Of these, 35 had no NR matches; these may be very rapidly evolving genes, genes in the late stages of degeneration, or the result of horizontal transfer from a genome not yet represented in the database [39]. It is also possible that some, especially the shorter of these genes, could be artefacts of the annotation process. Finally, nine $w$ Bol1b-specific genes lacked Wolbachia homologs but had high-quality matches to non-Wolbachia genes in the NR database. All but one of these genes are either within or adjacent to phage regions. We searched for degenerate or unannotated copies of these genes in the $w$ Pip genome and found no evidence of them, and it is likely that they represent recent phage-mediated horizontal gene transfers into the $w$ Bol1-b genome that occurred subsequent to divergence from $w$ Pip. These genes and their homologs are described below.

Two contiguous genes, wBol1_0262 and wBol1_0265, encode proteins with radical SAM ( $S$-adenosylmethionine) domains, which are known to play diverse molecular roles, including interaction between intracellular bacteria and their hosts $[40,41]$. These genes are divergent homologs of two contiguous genes in the genome of the Actinobacterium Micromonospora aurantiaca. The transposase gene wBol1_0093 has homologs in a diverse range of bacterial taxa, including the sponge symbiont Rhodobacteraceae bacterium KLH11 and the plant-associated environmental bacteria Burkholdaria cenocepacia and Dyadobacter fermentans, but there is insufficient phylogenetic resolution to determine which of these is most closely related to wBol1_0093 (Additional file 2: Figure S2a). wBol1_0035, which encodes a hypothetical protein, clusters with genes from the cyanobacterium Synechococcus sp CC9311 and the pathogen Legionella longbeachae (Additional file 2: Figure S2b), though with relatively low bootstrap support. wBol1_0187, also encoding a hypothetical protein, clusters with $100 \%$ bootstrap support with a gene from the methanogenic archaeon Methanococcoides burtonii (Additional file 2: Figure S2c). Divergent homologs of this gene are annotated in the genomes of A group strains, but these are distant from wBol1_0187 in the phylogenetic tree and form a separate, strongly supported clade with a gene from the homoacetogenic bacterium Clostridium ljungdahlii, suggesting two independent transfers into Wolbachia genomes.

The origin of two other contiguous $w$ Bol1-b-specific genes, wBol1_0256 and wBol1_0257, appears more complex. Both genes have as their top NR blastp match the Solenopsis invicta (fire ant) gene SINV_00084. The 5' and 3' ends of SINV_00084 are similar to fragments of insect Golgi SNAP receptor complex genes, while the highly internally repetitive central portion of the gene matches a region of the Rickettsia massiliae dnaE2 gene, RMA_0751, which is part of the tra cluster region [42]. This gene cluster, which encodes proteins involved in conjugal DNA transfer, is thought to have been laterally transferred into Rickettsia from Protochlamydia amoebophila, an obligate symbiont of amoebae [43]. Given the highly repetitive nature of the sequences involved, and the few taxa for which matching sequences are available in Genbank, it is not currently possible to determine the evolutionary history of the possible transfers of this genetic region between $w$ Bol1-b, $R$. massiliae, S. invicta or some number of possible intermediates not yet represented in the database. Finally, two additional $w$ Bol1-b genes, wBol1_1091 and wBol1_1092, discussed in further detail below, may have originated in eukaryotes.

What are the proximate sources of these horizontally transferred genes? There is clearly no single taxonomic group represented in the NR database that shares close homologs of this complete set of genes with $w$ Bol1-b. Furthermore, for several of these genes, the closest known homologs are moderately divergent from the copies in $w$ Bol1-b, and it is unlikely that the genetic 
transfer took place directly between these taxa and Wolbachia. These genes are found associated with different phage regions of the $w$ Boll-b genome, and may be the result of multiple independent transfers since divergence from $w$ Pip. Together, these observations suggest that the introduction of new genes into Wolbachia genomes is ongoing and frequent, and that the phage that mediate these transfers carry genetic material from an exceptionally diverse group of organisms.

\section{Horizontal gene transfer between wBol1-b and eukaryotes}

Two $w$ Bol1-b-specific genes that each contain a secA domain may have been transferred from eukaryotic rather than prokaryotic taxa. Proteins containing secA domains are best characterized in bacteria, where they act as ATPases mediating translocation of preproteins through the cytoplasmic membrane [44]. The core Wolbachia genome includes a copy of a typical bacterial secA (encoded by WD0549/WRi_003630/WPa_0368/ wBol1_0067/Wbm0266) that is of unexceptional length for Wolbachia genes (2604 nt) and has orthologs in closely related $\alpha$-Proteobacterial genera Ehrlichia and Anaplasma. The secA genes wBol1_1091 and wBol1_1092, however, show a different pattern. These two genes are unusually long for Wolbachia genes: wBol1_1091 is $4488 \mathrm{nt}$ in length and wBol1_1092 is 11,829 nt. Both have full-length matches in the NR database only to insect proteins.

wBol1_1091 has multiple full-length matches to hypothetical proteins in Culex quinquefasciatus and Aedes aegypti. The 5' half of the gene has matches only to the mosquito genes, but the 3 ' half of the gene, which contains the secA domain, also has further hits to basal eukaryotes, including Polysphondylium pallidum, Tetrahymena thermophila and Salpingoeca sp. There are no close bacterial matches. The highest-ranked bacterial match has low-level and fragmentary sequence similarity with a portion of the secA domain region of wBol1_1091, and has a bit score of only 108, compared to scores of 200 to 957 for the eukaryotic matches.

wBol1_1092 has full-length hits to multiple secA proteins in the jumping ant Harpegnathos saltator, and a near-full length hit to a hypothetical protein in Daphnia pulex. The region of the protein containing the secA domain has hits to hypothetical proteins in other insects including Drosophila willistoni, Culex quinquefasciatus and Tribolium castaneum. This region also has less significant hits to proteins from basal eukaryotes including Tetrahymena and Polysphondylium, and more distantly to bacterial secA proteins.

In the wBol1-b draft genome assembly, wBol1_1091 and wBol1_1092 are surrounded by known Wolbachia genes (Figure 3). We confirmed the position of these eukaryotic-like secA genes in the Wolbachia genome, and

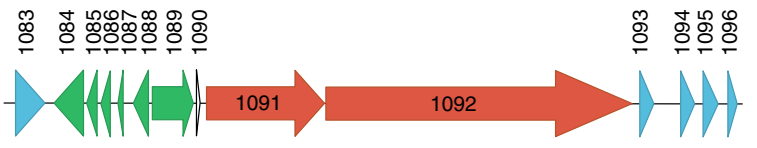

Figure 3 Location of horizontally transferred secA genes, wBol1_1091 and wBol1_1092, in the wBol1-b genome. Arrows represent CDSs, are drawn to scale, and are labelled with abbreviated locus names (e.g. WBol1_1091 is 1091). Core Wolbachia genes are shown in green, non-core genes with identifiable Wolbachia homologs are in blue.

their absence from uninfected host material, using PCRs spanning wBol1_1089-wBol1_1091 and wBol1_1092wBol1_1093, on infected and antibiotic-treated lines of H. bolina.

Other Wolbachia homologs of these secA genes were not found in complete genomes or in the NR database. However, matches were found to whole genome shotgun sequence data from the NC48S strain of Drosophila simulans, sequenced as part of the $D$. simulans genome project [45], when blasting against the NCBI WGS database. The NC48S line was collected in Noumea in 1991, and is superinfected with two Wolbachia strains, A group w $\mathrm{Ha}$ and $\mathrm{B}$ group $w$ No [46]. None of the other six lines of $D$. simulans sequenced for the $D$. simulans genome project, whether Wolbachia-uninfected or infected with $w \mathrm{Ri}$, appeared to contain sequence matches to these secA genes. To determine which of $w \mathrm{Ha}$ and $w$ No carries the secA homologs, we used NC48S lines in which the superinfection has been separated out into single infections. We amplified and sequenced portions of the homologs of both wBol1_1091 (516 nt) and wBol1_1092 (3018 nt) from the line infected with $w \mathrm{Ha}$, but could not amplify these regions in either the line infected with $w$ No or the antibiotic-treated uninfected lines. The sequences from $w$ Bol1-b and $w$ Ha were 93.4\% (wBol1_1091) and 94.6\% (wBol1_1092) identical at the nucleotide level. The secA genes from $w$ Bol1-b and $w$ Ha cluster together phylogenetically to the exclusion of their insect homologs (Additional file 2: Figure S3). wBol1_1092 is evolving under purifying selection in these two strains $(\omega=0.266 ; \omega<1$ with $P<0.001)$, suggesting that it is functional. wBol1_1091, however, appears to be evolving neutrally $(\omega=0.945 ; \omega$ not significantly $<1)$.

In what direction did the horizontal transfer of these genes between Wolbachia and insects occur? The taxonomic distribution of secA domain proteins in eukaryotes makes it clear that they have been susceptible to horizontal transfer between lineages. However, blastp searches using wBol1_1091 or wBol1_1092 as queries reveal a clear delineation between the full-length, high-similarity eukaryotic matches and lower-quality matches to bacteria, a pattern that is confirmed by phylogenetic analyses. The eukaryotic homologs of wBol1_1092 form a strongly supported clade separate from the prokaryotic homologs (Figure 4). Within 


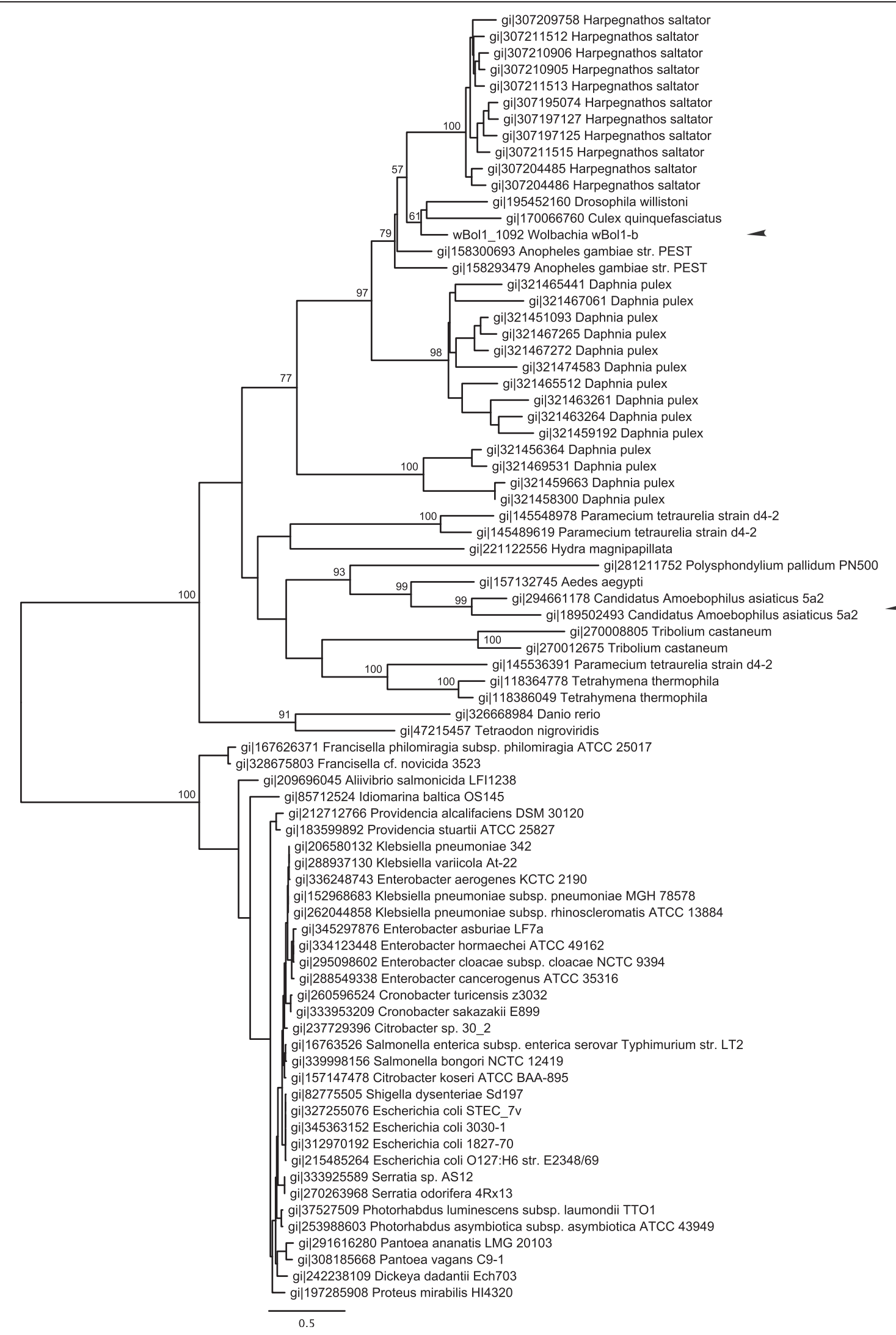

Figure 4 (See legend on next page.) 
the eukaryotic clade, there have been two transfers of this gene into bacteria: one represented by wBol1_1092, and the other by two Candidatus Amoebophilus asiaticus genes, Aasi_1610 and Aasi_1144, which cluster with an Aedes aegypti gene. Unlike wBol1_1092, however, these Candidatus Amoebophilus genes do not have full-length matches to insect secA proteins, with sequence similarity only in the secA domain.

The presence of introns in genes subject to interdomain horizontal gene transfer is often taken as evidence that transfer occurred from eukaryote to prokaryote. Although many of the eukaryotic secA genes are annotated with introns, most of these introns appear to be spurious (Additional file 2). There is little evidence of true introns in the eukaryotic secA genes most closely related to the Wolbachia genes, but this does not indicate that the transfer took place in the opposite direction: a substantial proportion of genes in these eukaryotic genomes are intronless, and there is no reason to believe that they all represent transfers from prokaryotes.

The extremely long gene length and phylogenetic analyses suggest that transfer of these genes occurred from eukaryotes into the Wolbachia genome. This matches the pattern of evidence observed for the gene WD0513, which was transferred between mosquito taxa and $w \mathrm{Mel} / w$ Pip $[47,48]$, and raises the possibility that Wolbachia genomes are able to receive, harbour, transfer, and possibly use protein coding genes of eukaryotic origin.

\section{Possible genomic bases of male killing}

Although male-killing bacteria have been described from diverse taxa, including Wolbachia, Spiroplasma, Rickettsia and Arsenophonous [17-20], little is known about the mechanism of male-killing in any of these systems. Spiroplasma poulsonii, the best characterized example to date, kills Drosophila melanogaster males only in the presence of all five peptides of the host dosage compensation complex [49], and this killing occurs during a narrow developmental period early in embryogenesis [50]. It has been speculated that male-killing in this system may occur through uncoordinated expression of the apoptosis pathway across the embryo, but the precise mechanism and the molecules secreted by Spiroplasma to initiate it are both unknown [50]. In contrast, Arsenophonous kills males in Nasonia by inhibiting the formation of maternally-inherited centrosomes, which are required for early male development [51]. The mechanisms of malekilling caused by strains of Wolbachia are generally less well characterized, and even more varied. Male-killing Wolbachia in D. bifasciata cause severe defects in chromatin remodelling and spindle organization in male embryos, leading to developmental failure [52]. The Wolbachia strain wSca feminizes males of its host, the moth Ostrinia scapulalis, and it is thought that it is a mismatch between the genetic and phenotypic sexes that causes male death [53]. The mechanism underlying male-killing by $w$ Bol1-b in $H$. bolina is uncharacterized, but it can act in both well-developed embryos and young larvae, suggesting that it is not due to specific targeting of an early developmental pathway in the host [54].

The diversity of mechanisms known to underlie malekilling in different systems makes it difficult to predict $a$ priori what the physiological or genetic basis of this phenotype is in $w$ Bol1-b. For this reason, using comparative genomics to identify candidate genes involved in male-killing is a valuable complementary approach to this question. Genes present in $w$ Bol1-b but absent from the genome of the closely related non-male-killing strain $w$ Pip are initial candidates for investigation. We have identified a number of genes specific to this genome (Additional file 2: Table S4), including functionally annotated single-copy genes, as well as genes coding for hypothetical proteins and paralogs of known Wolbachia genes, and these should be targets for further research.

It is also possible that the expression of male killing is mediated by additional factors, such as changes in the tropism or level of expression of genes that are also present in non-male-killing strains of Wolbachia. The availability of the $w$ Bol1-b genome will facilitate future transcriptomic analyses of expression patterns. Male killing could also potentially arise as a result of changes in gene sequence of common genes leading to modifications of their function or expression. We performed branch-specific and branch-site analyses of positive selection [55] to test for adaptive evolution in core genes along the lineage leading to $w$ Bol1-b, but there was insufficient power to identify significant changes in selection due to the short length of this branch of the phylogeny (data not shown). Finally, there are a number of studies showing that, for at least some strain-host combinations, the expression of male killing is hostdependent and that some hosts are able to evolve to 
repress male killing [56-58], and this should be taken into account in future investigations of the mechanism underlying this phenotype.

\section{Materials \& methods $w$ Bol1-b origin and transfer into cell culture}

A $w$ Bol1-b-infected butterfly (G0) was collected on the island of Moorea (French Polynesia) in February 2006. We dissected out the abdomen of a mature G1 $H$. bolina under aseptic conditions. The abdomen was surfacesterilized in $70 \%$ ethanol, then incised in a small volume of $96 \%$ ethanol. The eggs extracted from the abdomen were briefly surface-sterilized in 70\% ethanol. Approximately ten butterfly eggs were transferred into a $1.5 \mathrm{~mL}$ centrifuge tube and washed three times with $1 \mathrm{~mL}$ of a sucrose-phosphate-glutamate solution (SPG) [59], which briefly maintains Wolbachia viability outside host cells. Eggs were re-suspended in $1 \mathrm{~mL}$ SPG and crushed against the centrifuge tube walls using a micropestle.

Each well of a vial plate was filled sequentially with $200 \mu \mathrm{L}$ monolayers of Aedes albopictus RML12 cells (80\% confluent), $1 \mathrm{~mL}$ of Mitsuhashi and Maramorosh insect medium [60] and $500 \mu \mathrm{L}$ of the egg extract. The plate was centrifuged at $800 \times \mathrm{g}$ for 1 hour, then incubated overnight at $26^{\circ} \mathrm{C}$. Cells were re-suspended and added to $5 \mathrm{~mL}$ of fresh insect cell culture medium in a $20 \mathrm{~cm}^{2}$ flask, and then maintained in-vitro by transfer into fresh medium as per normal every four days [61].

\section{Wolbachia DNA preparation}

We grew wBol1-b-infected cells for 6-15 weeks after the transfer of the infection to RML12 cell culture. From a total of 500 cell culture flasks $\left(175 \mathrm{~cm}^{2}\right)$, six $w$ Bol1-b DNA samples were purified using the protocol described by [26], followed by an extra separation step on percoll gradient. Six Beckman ultra-clear centrifuge tubes $\left(9 / 16 \times 3 \frac{1}{2}\right.$ inch, $14 \times 89 \mathrm{~mm}$ ), each containing four density layers (from bottom to top: $2 \mathrm{~mL}$ of $60 \%$ Percoll, $4 \mathrm{~mL}$ of $40 \%$ Percoll, $3 \mathrm{~mL}$ of $20 \%$ Percoll and $2.7 \mathrm{~mL}$ of $10 \%$ Percoll) were prepared on ice. The gradients were loaded with $700 \mu \mathrm{L}$ sample and placed in the swinging SW41 rotor buckets of a Beckman Optimal-L-80 XP ultracentrifuge and centrifuged for one hour at $10,900 \mathrm{xg}$ at $4^{\circ} \mathrm{C}$. This step separates Wolbachia cells from other cell components and debris based on their density, and four opaque bands of cellular material appeared between the different Percoll layers. The bottom opaque band (band \#4) between the $40 \%$ and $60 \%$ Percoll layers was collected.

DNA from G0 butterfly, G1 butterfly and transinfected cells was extracted using a Qiagen DNAeasy blood and tissue extraction kit. The $w$ Boll-b infection status of the samples was confirmed by PCR with $w$ Bol1-b specific primers $81 \mathrm{~F} / 522 \mathrm{R}$ and $\mathrm{Gp} 1 \mathrm{bF} / \mathrm{R}$ [15] respectively, Additional file 2: Table S2, [62]. The DNA composition of band \#4 of the Percoll density gradient was also characterized by PCR, after extracting the DNA from an aliquot using phenol-chloroform. Presence of Wolbachia strain $w$ Bol1-b DNA was confirmed by PCR amplification of the surface protein gene $w s p b$, using primers $81 \mathrm{~F} /$ 522R, and the ankyrin gene WD637, using primers $693 \mathrm{~F} /$ 693R. Primer pairs for the Aedes aegypti 18S rRNA gene and protein-coding genes RPS7 and EF (18S-F/18S-R, AgRPS7-F/AgRPS-R and EF-F/EF-R) amplified Aedes albopictus contaminating DNA. The12S rRNA gene was used as a mitochondrial DNA marker. The purification quality was assessed by running $1 \mu \mathrm{g}$ of the Wolbachia DNA in a $1 \%$ agarose gel for $40 \mathrm{~min}$, with $1 \mathrm{~kb}$ DNA ladder.

\section{wBol1-b genome sequencing and assembly}

Approximately $5 \mu \mathrm{g}$ of Wolbachia DNA was provided to AGRF (Australian Genome Research Facility), which generated a 454 GS-FLX shotgun library. An additional sample was then provided to AGRF to generate a 454 GS-FLX paired-ends library. Libraries were combined for genome assembly.

Genome assembly was done by AGRF following the protocol provided by the platform supplier (Roche) and based on a shotgun-data-first addition order. Contaminating mosquito sequence was identified by using contigs as blastN queries against the NR database and identifying high-similarity matches. The assembly was manually edited using Artemis [63]. The final assembly consists of 91 contigs, ranging from 644 bp to 155817 bp in length, arranged into 13 scaffolds, and an additional 53 unscaffolded contigs with lengths less than $2 \mathrm{~kb}$. The sequence data has been deposited at the European Nucleotide Archive (CAOH01000001-CAOH01000144) in BioProject PRJEB566.

We confirmed that the strain sequenced was a clonal lineage by mapping the reads to the assembly using Newbler v2.6 (Roche) and checking for evidence of wellsupported SNPs, which would suggest that a mixed culture had been sequenced. All high-confidence SNPs were associated with known imperfect repeats (data not shown), indicating that the strain sequenced is highly likely to have been clonal.

\section{Annotation}

Initial annotation of the $w$ Bol1-b draft genome assembly was performed using SUGAR (Simple Unfinished Genome Annotation Resource), an annotation pipeline consisting of several Perl scripts, controlled by a user defined instruction file (Szubert \& Beatson, In Prep.). The program makes use of the NUCmer component of the MUMmer 3.0 package [64] for ordering an unfinished genome against at least one reference sequence. Glimmer 3.02 [65] was used for protein coding gene 
calling (after punctuating contig boundaries with a six frame stop-start sequence), based on a set of observed long ORFs, with optional scanning for genes matching over boundaries, and improvements to paired ends derived scaffolding. Automated annotation of proteins was based on a diminishing identity threshold scale for Blastp [66] matches against protein databases consisting of (1) the reference genome wPip, (2) other Wolbachia genomes, (3) swiss-prot and (4) the non-redundant database (NR). Annotations based on profile matches in Pfam [67], TIGRFAM [68] and COG [69] databases were also supplied. t-RNA genes were predicted using TE-SCAN [70].

\section{Ortholog prediction}

We performed an all-versus-all blastp analysis of the predicted proteomes of $w \mathrm{Mel}, w \mathrm{Ri}, w \mathrm{Pip}, w \mathrm{Bol1}-\mathrm{b}$ and $w \mathrm{Bm}$, then used orthoMCL [27] to group orthologs and their recent paralogs (lineage-specific duplications) into 'ortholog clusters'. We ran the analysis multiple times using E-value cut-offs of 1e-05 and 1e-10, and with inflation values of 1.2, 1.5, 2.5 and 5 . The great majority of core ortholog clusters were identical across runs; we performed all further analyses on results of the run with default settings (E-value 1e-5, inflation 1.5). We used purpose-written Perl scripts to parse the results of this analysis and identify clusters of core genes. To confirm that the ortholog clustering was reasonable, we compared the core gene ortholog sets produced by orthoMCL to those predicted using two other methods: a simple all-versus-all mutual best blast hit analysis, and the sets of single-copy positional homologs inferred by Mauve [71] after alignment of the five genome sequences. 659 core ortholog sets were predicted by at least one of the three methods. Of these, 577 (88\%) were predicted by all three, 67 (10\%) by two, and $15(2 \%)$ by one method. For the 67 ortholog sets predicted by two methods, 65 of them were predicted by orthoMCL and mutual best blast hits, but not by Mauve. Many of the ortholog sets not called by Mauve had substantial differences in gene length between orthologs, or synteny breaks adjacent to one or more orthologs in the genome alignment, which were generally sufficient to explain the difference in predictions. We manually inspected all ortholog sets predicted by one method only, and approximately $50 \%$ of the sets predicted by two methods. In almost all cases, the orthoMCL prediction was supported by inspection.

\section{wBol1-b-specific genes}

To identify genes specific to $w$ Boll-b, we used the amino acid sequences of all of the $w$ Bol1-b genes that were not clustered with any other genes in the orthoMCL analysis as blastp queries against the NR database with a very low stringency E-value threshold of 10. Genes were considered putatively wBol1-b-specific if they had either no hits to the NR database with this cut-off, or had no hit to any Wolbachia gene with a better E-value than the best hit to a non-Wolbachia gene.

\section{Phylogenetic analyses}

For the MLST tree, we manually aligned nucleotide sequences of the $\operatorname{cox} A, f b p A, f t s Z$, gat $B$ and $h c p A$ genes [72] from $w$ Mel, $w$ Ri, $w$ Pip, $w$ Bol1-a, $w$ Bol1-b, $w$ Bol2 and $w \mathrm{Bm}$, obtained from Genbank, then concatenated the alignments. We inferred a phylogenetic tree using PHYML [73], using the HKY substitution matrix, a discrete gamma model with four rate classes and a gamma shape parameter estimated from the data. For the phylogenetic network, we used t_coffee [74] to align sequences of the 654 core genes from the five Wolbachia genomes. These single-gene alignments were concatenated to form an alignment $681,717 \mathrm{nt}$ in length. A Neighbor-Net network [33] was inferred from this alignment using default parameter values in SplitsTree [75]. For the phylogenetic analyses of secA and other putatively horizontally transferred genes, we aligned amino acid sequences using t_coffee [74], then edited and trimmed alignments by eye. We inferred phylogenetic trees using PHYML [73], using the JTT substitution matrix and four substitution rate classes with the gamma parameter estimated from the data.

\section{Synteny analysis}

Synteny between the $w$ Bol1-b assembly and other complete Wolbachia genomes was visualised using NUCmer (with parameter settings - -maxgap $=500,-$ mincluster $=100)$ and mummerplot [64].

\section{wBol1-b WO prophage regions}

Putative prophage regions were identified using a combination of two methods. First, the $w$ Pip, $w$ CauB and $w$ VitB $W O$ prophage proteins (as defined in [36]) were used as blastp queries against the $w$ Bol1-b protein sequences. Secondly, we identified $w$ Bol1-b genes that clustered with $w$ Pip prophage genes in the orthoMCL results. Boundaries of the putative prophage regions were determined by a combination of checking homology with $w$ Pip and $w$ CauB prophages and manual assessment of gene annotations in boundary regions.

\section{Sequencing of secA genes}

The presence and position of secA genes in $w$ Bol1-b was confirmed by sequencing PCR products obtained using primers spanning the wBol1-b_1089-wBol1-b_1091 boundary (mutLSecA1-F + mutLSecA1-R) and the wBol1-b_1092wBol1-b_1093 boundary (SecA2Tran-F + SecA2 Tran-R). The wBol1-b_1092 ortholog in wHa was amplified using 
various combinations of primers 1092-2, -3, -4, -5, -6, which were designed based on the $w \mathrm{Ha}$ sequence fragments present in the NCBI WGS database. The wBol1b_1091 $w \mathrm{Ha}$ ortholog was amplified using primers 1091-F and 1091-R, also based on $w \mathrm{Ha}$ sequence. Primer sequences are listed in Additional file 2: Table S2. PCR cycling conditions were as follows: $94^{\circ} \mathrm{C} 3 \mathrm{~min},\left(94^{\circ} \mathrm{C} 30 \mathrm{~s}\right.$, $\left.52^{\circ} \mathrm{C} 30 \mathrm{~s}, 68^{\circ} \mathrm{C} 150 \mathrm{~s}\right) \times 35$ cycles, then $68^{\circ} \mathrm{C} 10 \mathrm{~min}$. The reaction mixture contained $625 \mathrm{nM}$ of each primer, $125 \mu \mathrm{M}$ dNTPs, $1.5 \mathrm{mM} \mathrm{MgSO} 4,20 \mathrm{ng}$ of DNA and $0.5 \mu \mathrm{L}$ of proof-reading Elongase enzyme mix (Invitrogen) in a final volume of $25 \mu \mathrm{l}$. PCR products were separated in $1 \%$ agarose gels and stained with ethidium bromide. Purified PCR products were sequenced at the Micromon facility (Monash University, Clayton, Australia) to confirm the insertion into the Wolbachia genome and the identity of the sequences.

\section{Selection analyses}

To test whether genes were evolving under purifying selection, we used codeml [55]. We used likelihood ratio tests (LRT) first to confirm that there was no significant variation in $\omega$ values between lineages, and then to compare the likelihood of a model of evolution that fixed $\omega=1$ with that of a model that allowed $\omega$ to be estimated from the data. If the latter LRT was significant, we checked that the estimated $\omega$ was $<1$.

\section{Additional files}

Additional file 1: Table S1. Clusters.xls, which lists ortholog clusters of core genes as identified by orthoMCL.

Additional file 2: Figure S1. Establishment of cell culture; Table S2. primers used in this study; Table S3. partial prophage regions in wBol1-b; Table S4. wBol1-b-specific genes; Figure S2. phylogenetic trees of horizontally transferred genes; Figure S3. phylogenetic tree of wBol1_1092 including partial $w \mathrm{Ha}$ homolog; Introns in eukaryotic secA genes; Note on annotation of WD1302.

\section{Competing interests}

The authors declare that they have no competing interests.

\section{Authors' contributions}

$\mathrm{SO}, \mathrm{AD}, \mathrm{MW}, \| \mathrm{O}, \mathrm{JB}, \mathrm{EM}, \mathrm{GH}$ and $\mathrm{SC}$ participated in the design and oversight of the study. AD, $\| O$ and $C M$ carried out laboratory work. SB, JS, AD and MW annotated the genome. $\mathrm{AD}$ and $\mathrm{MW}$ analysed the data and drafted the manuscript. All authors contributed to and approved the final manuscript.

\section{Acknowledgements}

We would like to thank B. Cass, J. Howie, M. Sidhu and Y-S. Leong for their help in the lab. We are also extremely grateful to A. Men and D. Miller from AGRF and to A. Darby for advice on WBol1-b DNA preparation prior to whole genome sequencing. We thank T. Walker and L. Klasson for constructive discussions, and K. Bourtzis and D. Cordeaux for examining and commenting on a previous draft of this study. Financial Support was provided by the Australian Research Council (DP0772992) grant awarded to SLO, the Genetics Society (Heredity Fieldwork Grants) and Sigma Xi (Grant in Aid of Research) grants awarded to AD, and NSF (grant-0416268) and NERC (grant-NE/ B503292/1) grants awarded to SC and GDDH

\section{Author details}

${ }^{1}$ School of Biological Sciences, University of Queensland, 4072, Brisbane, QLD, Australia. ${ }^{2}$ Metapopulation Research Group, The University of Helsinki, PO Box 65Viikinkaari 1, 00014, Helsinki, Finland. ${ }^{3}$ School of Biological Sciences, Monash University, Clayton, VIC 3800, Australia. ${ }^{4}$ School of Chemistry and Molecular Biosciences, University of Queensland, Brisbane, QLD 4072, Australia. ${ }^{5}$ School of Biomolecular and Physical Sciences, Griffith University, Brisbane 4111, Australia. ' Laboratory of Neurogenetics and Behavior, The Rockefeller University, 1230 York Avenue, Campus Box 63, New York, NY 10065, USA. 'Institute of Integrative Biology, University of Liverpool, Biosciences Building, Crown Street, Liverpool L69 7ZB, United Kingdom. ${ }^{8}$ UMR CNRS 5558, Laboratoire de Biométrie et Biologie Évolutive, UCB Lyon 1, Bâtiment Grégor Mendel, 43 bd du 11 novembre 1918, 69622,

Villeurbanne cedex, France. ${ }^{9}$ Institute for Molecular Bioscience, University of Queensland, 4072, Brisbane, QLD, Australia.

Received: 27 August 2012 Accepted: 2 January 2013

Published: 16 January 2013

\section{References}

1. Hilgenboecker $K$, Hammerstein P, Schlattmann P, Telschow A, Werren JH: How many species are infected with Wolbachia? - a statistical analysis of current data. FEMS Microbiol Lett 2008, 281(2):215-220.

2. Werren $\mathrm{JH}$, Baldo L, Clark ME: Wolbachia: master manipulators of invertebrate biology. Nat Rev Microbiol 2008, 6(10):741-751.

3. Bandi C, Anderson TJC, Genchi C, Blaxter ML: Phylogeny of Wolbachia in filarial nematodes. Proc R Soc London, Ser B 1998, 265(1413):2407-2413.

4. Dobson SL, Bourtzis K, Braig HR, Jones BF, Zhou WG, Rousset F, O'Neill SL: Wolbachia infections are distributed throughout insect somatic and germ line tissues. Insect Biochem Mol Biol 1999, 29(2):153-160.

5. Brownlie JC, Cass BN, Riegler M, Witsenburg JJ, Iturbe-Ormaetxe I, McGraw EA, O'Neill SL: Evidence for metabolic provisioning by a common invertebrate endosymbiont, Wolbachia pipientis, during periods of nutritional stress. PLoS Pathog 2009, 5(4):e1000368.

6. Hedges LM, Brownlie JC, O'Neill SL, Johnson KN: Wolbachia and virus protection in insects. Science 2008, 322(5902):702-702.

7. Moreira LA, Iturbe-Ormaetxe I, Jeffery JA, Lu GJ, Pyke AT, Hedges LM, Rocha BC, Hall-Mendelin S, Day A, Riegler M, et al: A Wolbachia symbiont in Aedes aegypti limits infection with dengue, chikungunya, and Plasmodium. Cell 2009, 139(7):1268-1278.

8. Teixeira L, Ferreira A, Ashburner M: The bacterial symbiont Wolbachia induces resistance to RNA viral infections in Drosophila melanogaster. PLOS Biol 2008, 6(12):2753-2763.

9. Walker $\mathrm{T}$, Johnson PH, Moreira LA, Iturbe-Ormaetxe I, Frentiu FD, McMeniman CJ, Leong YS, Dong Y, Axford J, Kriesner P, et al: The wMel Wolbachia strain blocks dengue and invades caged Aedes aegypti populations. Nature 2011, 476(7361):450-U101.

10. Wu M, Sun LV, Vamathevan J, Riegler M, Deboy R, Brownlie JC, McGraw EA, Martin W, Esser C, Ahmadinejad N, et al: Phylogenomics of the reproductive parasite Wolbachia pipientis wMel: A streamlined genome overrun by mobile genetic elements. PLoS Biol 2004, 2(3):327-341.

11. Klasson L, Westberg J, Sapountzis P, Nasiund $K$, Lutnaes $Y$, Darby AC, Veneti $Z$, Chen LM, Braig HR, Garrett R, et al: The mosaic genome structure of the Wolbachia wRi strain infecting Drosophila simulans. PNAS 2009, 106(14):5725-5730.

12. Klasson L, Walker T, Sebaihia M, Sanders MJ, Quail MA, Lord A, Sanders S, Earl J, O'Neill SL, Thomson N, et al: Genome evolution of Wolbachia strain wPip from the Culex pipiens group. Mol Biol Evol 2008, 25:1877-1887.

13. Foster J, Ganatra M, Kamal I, Ware J, Makarova K, Ivanova N, Bhattacharyya A, Kapatral V, Kumar S, Posfai J, et al: The Wolbachia genome of Brugia malayi: Endosymbiont evolution within a human pathogenic nematode. PLOS Biol 2005, 3(4):599-614.

14. Augustinos AA, Santos-Garcia D, Dionyssopoulou E, Moreira M, Papapanagiotou A, Scarvelakis M, Doudoumis V, Ramos S, Aguiar AF, Borges PAV, et al: Detection and characterization of Wolbachia infections in natural populations of aphids: Is the hidden diversity fully unraveled? PLoS One 2011, 6(12):11.

15. Zhou WG, Rousset F, O'Neill S: Phylogeny and PCR-based classification of Wolbachia strains using wsp gene sequences. Proc $R$ Soc London, Ser B 1998, 265(1395):509-515. 
16. Ishmael N, Hotopp JCD, loannidis P, Biber S, Sakamoto J, Siozios S, Nene V, Werren J, Bourtzis K, Bordenstein SR, et al: Extensive genomic diversity of closely related Wolbachia strains. Microbiol-(UK) 2009, 155:2211-2222.

17. Gherna RL, Werren JH, Weisburg W, Cote R, Woese CR, Mandelco L, Brenner $D J$ : Arsenophonus nasoniae gen. nov., sp. nov., the causative agent of the son-killer trait in the parasitic wasp Nasonia vitripennis. Int J Syst Bacteriol 1991, 41(4):563-565.

18. Haselkorn TS: The Spiroplasma heritable bacterial endosymbiont of Drosophila. Fly 2010, 4(1):80-87.

19. Hurst GDD, Jiggins FM, von der Schulenburg JHG, Bertrand D, West SA, Goriacheva II, Zakharov IA, Werren JH, Stouthamer R, Majerus MEN: Malekilling Wolbachia in two species of insect. Proc R Soc London, Ser B 1999, 266(1420):735-740.

20. Werren JH, Hurst GDD, Zhang W, Breeuwer JAJ, Stouthamer R, Majerus MEN Rickettsial relative associated with male killing in the ladybird beetle (Adalia bipunctata). J Bacteriol 1994, 176(2):388-394.

21. Charlat S, Hornett EA, Dyson EA, Ho PPY, Loc NT, Schilthuizen M, Davies N, Roderick GK, Hurst GDD: Prevalence and penetrance variation of malekilling Wolbachia across Indo-Pacific populations of the butterfly Hypolimnas bolina. Mol Ecol 2005, 14(11):3525-3530.

22. Charlat S, Reuter M, Dyson EA, Hornett EA, Duplouy A, Davies N, Roderick GK, Wedell N, Hurst GDD: Male-killing bacteria trigger a cycle of increasing male fatigue and female promiscuity. Curr Biol 2007, 17(3):273-277.

23. Hornett EA, Charlat S, Duplouy AMR, Davies N, Roderick GK, Wedell N, Hurst GDD: Evolution of male-killer suppression in a natural population. PLOS Biol 2006, 4(9):1643-1648.

24. Hornett EA, Duplouy AMR, Davies N, Roderick GK, Wedell N, Hurst GDD, Charlat S: You can't keep a good parasite down: Evolution of a male-killer suppressor uncovers cytoplasmic incompatibility. Evolution 2008, 62(5):1258-1263.

25. Darby AC, Choi JH, Wilkes T, Hughes MA, Werren JH, Hurst GDD, Colbourne $\mathrm{JK}$ : Characteristics of the genome of Arsenophonus nasoniae, son-killer bacterium of the wasp Nasonia. Insect Mol Biol 2010, 19:75-89.

26. Iturbe-Ormaetxe I, Woolfit M, Rances E, Duplouy A, O'Neill SL: A simple protocol to obtain highly pure Wolbachia endosymbiont DNA for genome sequencing. J Microbiol Meth 2011, 84(1):134-136.

27. Li L, Stoeckert CJ, Roos DS: OrthoMCL: Identification of ortholog groups for eukaryotic genomes. Genome Res 2003, 13(9):2178-2189.

28. Pichon S, Bouchon D, Cordaux R, Chen LM, Garrett RA, Greve P. Conservation of the type IV secretion system throughout Wolbachia evolution. Biochem Biophys Res Commun 2009, 385(4):557-562.

29. Iturbe-Ormaetxe I, Burke GR, Riegler M, O'Neill SL: Distribution, expression, and motif variability of ankyrin domain genes in Wolbachia pipientis. J Bacteriol 2005, 187(15):5136-5145.

30. Walker T, Klasson L, Sebaihia M, Sanders MJ, Thomson NR, Parkhill J, Sinkins SP: Ankyrin repeat domain-encoding genes in the wPip strain of Wolbachia from the Culex pipiens group. BMC Biol 2007, 5:39.

31. Pan XX, Luhrmann A, Satoh A, Laskowski-Arce MA, Roy CR: Ankyrin repeat proteins comprise a diverse family of bacterial type IV effectors. Science 2008, 320(5883):1651-1654

32. Papafotiou G, Oehler S, Savakis C, Bourtzis K: Regulation of Wolbachia ankyrin domain encoding genes in Drosophila gonads. Res Microbiol 2011 162(8):764-772.

33. Bryant $D$, Moulton $V$ : Neighbor-Net: An agglomerative method for the construction of phylogenetic networks. Mol Biol Evol 2004, 21(2):255-265.

34. Cordaux R, Pichon S, Ling A, Perez P, Delaunay C, Vavre F, Bouchon D, Greve $P$ : Intense transpositional activity of insertion sequences in an ancient obligate endosymbiont. Mol Biol Evol 2008, 25(9):1889-1896.

35. Felsheim RF, Kurtti TJ, Munderloh UG: Genome sequence of the endosymbiont Rickettsia peacockii and comparison with virulent Rickettsia rickettsii: identification of virulence factors. PLoS One 2009, 4(12):e8361.

36. Kent BN, Funkhouser LJ, Setia S, Bordenstein SR: Evolutionary genomics of a temperate bacteriophage in an obligate intracellular bacteria (Wolbachia). PLoS One 2011, 6(9):e24984.

37. Bordenstein SR, Wernegreen JJ: Bacteriophage flux in endosymbionts (Wolbachia): Infection frequency, lateral transfer, and recombination rates. Mol Biol Evol 2004, 21(10):1981-1991.

38. Kent BN, Salichos L, Gibbons JG, Rokas A, Newton ILG, Clark ME, Bordenstein SR: Complete bacteriophage transfer in a bacterial endosymbiont (Wolbachia) determined by targeted genome capture. Genome Biol Evol 2011, 3:209-218.

39. Daubin V, Ochman H: Bacterial genomes as new gene homes: the genealogy of ORFans in E. coli. Genome Res 2004, 14(6):1036-1042.
40. Sofia HJ, Chen G, Hetzler BG, Reyes-Spindola JF, Miller NE: Radical SAM, a novel protein superfamily linking unresolved steps in familiar biosynthetic pathways with radical mechanisms: functional characterization using new analysis and information visualization methods. Nucleic Acids Res 2001, 29(5):1097-1106.

41. Choi JY, Lee TW, Jeon KW, Ahn TI: Evidence for symbiont-induced alteration of a host's gene expression: Irreversible loss of SAM synthetase from Amoeba proteus. J Eukaryot Microbiol 1997, 44(5):412-419.

42. Blanc G, Ogata H, Robert C, Audic S, Claverie JM, Raoult D: Lateral gene transfer between obligate intracellular bacteria: Evidence from the Rickettsia massiliae genome. Genome Res 2007, 17(11):1657-1664.

43. Ogata H, La Scola B, Audic S, Renesto P, Blanc G, Robert C, Fournier PE, Claverie JM, Raoult D: Genome sequence of Rickettsia bellii illuminates the role of amoebae in gene exchanges between intracellular pathogens. PLoS Genet 2006, 2(5):733-744.

44. Manting EH, Driessen AJM: Escherichia coli translocase: the unravelling of a molecular machine. Mol Microbiol 2000, 37(2):226-238.

45. Begun DJ, Holloway AK, Stevens K, Hillier LW, Poh YP, Hahn MW, Nista PM, Jones CD, Kern AD, Dewey CN, et al: Population genomics: Wholegenome analysis of polymorphism and divergence in Drosophila simulans. PLoS Biol 2007, 5(11):2534-2559.

46. James AC, Dean MD, MCMahon ME, Ballard JWO: Dynamics of double and single Wolbachia infections in Drosophila simulans from New Caledonia. Heredity 2002, 88:182-189.

47. Klasson L, Kambris Z, Cook PE, Walker T, Sinkins SP: Horizontal gene transfer between Wolbachia and the mosquito Aedes aegypti. BMC Genomics 2009, 10:33.

48. Woolfit M, Iturbe-Ormaetxe I, McGraw EA, O'Neill SL: An ancient horizontal gene transfer between mosquito and the endosymbiotic bacterium Wolbachia pipientis. Mol Biol Evol 2009, 26(2):367-374.

49. Veneti Z, Bentley JK, Koana T, Braig HR, Hurst GDD: A functional dosage compensation complex required for male killing in Drosophila. Science 2005, 307(5714):1461-1463.

50. Bentley JK, Veneti Z, Heraty J, Hurst GDD: The pathology of embryo death caused by the male-killing Spiroplasma bacterium in Drosophila nebulosa. BMC Biol 2007, 5:9.

51. Ferree PM, Avery A, Azpurua J, Wilkes T, Werren JH: A bacterium targets maternally inherited centrosomes to kill males in Nasonia. Curr Biol 2008, 18(18):1409-1414.

52. Riparbelli MG, Giordano R, Ueyama M, Callaini G: Wolbachia-mediated male killing is associated with defective chromatin remodeling. PLoS One 2012, 7(1):e30045.

53. Sugimoto TN, Fujii T, Kayukawa T, Sakamoto H, Ishikawa Y: Expression of a doublesex homologue is altered in sexual mosaics of Ostrinia scapulalis moths infected with Wolbachia. Insect Biochem Mol Biol 2012, 40(12):847-854.

54. Charlat S, Davies N, Roderick GK, Hurst GDD: Disrupting the timing of Wolbachia-induced male-killing. Biol Lett 2007, 3(2):154-156.

55. Yang ZH: PAML: a program package for phylogenetic analysis by maximum likelihood. Comput Appl Biosci 1997, 13(5):555-556.

56. Hornett EA, Charlat S, Wedell N, Jiggins CD, Hurst GDD: Rapidly shifting sex ratio across a species range. Curr Biol 2009, 19(19):1628-1631.

57. Hornett EA, Engelstadter J, Hurst GDD: Hidden cytoplasmic incompatibility alters the dynamics of male-killer/host interactions. J Evol Biol 2010, 23(3):479-487.

58. Jaenike J: Spontaneous emergence of a new Wolbachia phenotype. Evolution 2007, 61(9):2244-2252.

59. Xi ZY, Dobson SL: Characterization of Wolbachia transfection efficiency by using microinjection of embryonic cytoplasm and embryo homogenate. Appl Environ Microbiol 2005, 71(6):3199-3204.

60. Mitsuhashi J, Maramorosch K: Leafhopper tissue culture: embryonic, nymphal, and imaginal tissues from aseptic insects. Contrib Boyce Thompson Inst 1964, 22:435-460.

61. McMeniman CJ, Lane AM, Fong AWC, Voronin DA, Iturbe-Ormaetxe I, Yamada R, McGraw EA, O'Neill SL: Host adaptation of a Wolbachia strain after long-term serial passage in mosquito cell lines. Appl Environ Microbiol 2008, 74(22):6963-6969.

62. Charlat S, Duplouy A, Hornett EA, Dyson EA, Davies N, Roderick GK, Wedell N, Hurst GDD: The joint evolutionary histories of Wolbachia and mitochondria in Hypolimnas bolina. BMC Evol Biol 2009, 9:64.

63. Carver T, Berriman M, Tivey A, Patel C, Bohme U, Barrell BG, Parkhill J, Rajandream MA: Artemis and ACT: viewing, annotating and comparing sequences stored in a relational database. Bioinformatics 2008, 24(23):2672-2676. 
64. Kurtz S, Phillippy A, Delcher AL, Smoot M, Shumway M, Antonescu C, Salzberg SL: Versatile and open software for comparing large genomes. Genome Biol 2004, 5(2):R12.

65. Delcher AL, Harmon D, Kasif S, White O, Salzberg SL: Improved microbial gene identification with GLIMMER. Nucleic Acids Res 1999, 27(23):4636-4641.

66. Altschul SF, Gish W, Miller W, Myers EW, Lipman DJ: Basic local alignment search tool. J Mol Biol 1990, 215(3):403-410.

67. Finn RD, Tate J, Mistry J, Coggill PC, Sammut SJ, Hotz HR, Ceric G, Forslund K, Eddy SR, Sonnhammer ELL, et al: The Pfam protein families database. Nucleic Acids Res 2008, 36:D281-D288.

68. Haft DH, Selengut JD, White O: The TIGRFAMs database of protein families. Nucleic Acids Res 2003, 31(1):371-373.

69. Tatusov RL, Koonin EV, Lipman DJ: A genomic perspective on protein families. Science 1997, 278(5338):631-637.

70. Lowe TM, Eddy SR: tRNAscan-SE: A program for improved detection of transfer RNA genes in genomic sequence. Nucleic Acids Res 1997, 25(5):955-964.

71. Darling ACE, Mau B, Blattner FR, Perna NT: Mauve: Multiple alignment of conserved genomic sequence with rearrangements. Genome Res 2004, 14(7):1394-1403.

72. Baldo L, Hotopp JCD, Jolley KA, Bordenstein SR, Biber SA, Choudhury RR, Hayashi C, Maiden MCJ, Tettelin H, Werren JH: Multilocus sequence typing system for the endosymbiont Wolbachia pipientis. Appl Environ Microbiol 2006, 72(11):7098-7110.

73. Guindon S, Gascuel O: A simple, fast, and accurate algorithm to estimate large phylogenies by maximum likelihood. Syst Biol 2003, 52(5):696-704.

74. Notredame C, Higgins DG, Heringa J: T-Coffee: A novel method for fast and accurate multiple sequence alignment. J Mol Biol 2000, 302(1):205-217.

75. Huson DH, Bryant D: Application of phylogenetic networks in evolutionary studies. Mol Biol Evol 2006, 23(2):254-267.

doi:10.1186/1471-2164-14-20

Cite this article as: Duplouy et al:: Draft genome sequence of the malekilling Wolbachia strain $w B$ Bol 1 reveals recent horizontal gene transfers from diverse sources. BMC Genomics 2013 14:20.

\section{Submit your next manuscript to BioMed Central and take full advantage of:}

- Convenient online submission

- Thorough peer review

- No space constraints or color figure charges

- Immediate publication on acceptance

- Inclusion in PubMed, CAS, Scopus and Google Scholar

- Research which is freely available for redistribution 